\title{
Toward Web-based Careflow Management Systems
}

\author{
Keith Miller and Wendy MacCaull \\ Centre for Logic and Information \\ St. Francis Xavier University \\ Antigonish, NS B2G 2W5 \\ Email: \{kmiller,wmaccaul\}@stfx.ca
}

\begin{abstract}
Health care systems are prime examples of ultra large scale systems involving complex, distributive processes with a high degree of variability. There are ubiquitous communication and massive data and knowledge management requirements including documentation and reporting. Health care systems are also critical systems, where errors can be very costly in terms of lives, quality of life, and/or dollars. The efficient use of limited resources is not only desirable but necessary. Designing these processes and managing their performance is difficult and error prone. We discuss a web-based Careflow Management System, currently under development, that takes advantage of emerging web technology and extends existing workflow management systems with formal verification features applying high performance computing methods to support real-time monitoring and adaptation. Healthcare ontologies are integrated into the system to allow advanced reasoning and to ensure accurate and relevant knowledge sharing among the various collaborators enhancing interoperability between specialized systems devoted to each area.
\end{abstract}

Index Terms-web-based systems, ontology, interoperability, verification, healthcare

\section{INTRODUCTION}

Healthcare systems are prime examples of "ultra large scale systems" where information and services are distributed, data is largely unstructured and unmanaged, processes are complex and evolving, errors are expensive and compromise safety, and computations are often complex. As an added twist - implementations require a high degree of flexibility to deal with inherent variability. While there are many technology-related successes in the health/medical field, capacity for process, information, and communication management is critically lacking. Innovative software to integrate and organize data, to provide real time intelligent process and information management, to provide quality assurance, and to adapt processes to changing conditions is critical.

Consider a typical scenario in a healthcare setting: A patient, Bertha, has cancer. She is confused, has issues with pain and needs assistance in the home. She will need:

- $\quad$ Assessments: pain, home care etc;

Keith Miller (kmiller@stfx.ca) is supported by a Science and Engineering Research Council of Canada Industrial Postgraduate Scholarship Wendy MacCaull (wmaccaul@stfx.ca) is supported by the Science and Engineering Research Council of Canada and the Atlantic Canada Opportunities Agency
- $\quad$ Procedures: chemotherapy, blood transfusions;

- Frequent tests at the lab;

- Therapy: various people/various locations;

- Medications prescribed by various physicians;

- Periodic reevaluation: every 3- 4 weeks or as often as necessary;

- $\quad$ Education and support for herself and her family.

There are simple dependencies among the tasks; some are required before or after others while some tasks may be done in parallel and other tasks are repeated periodically. There are also more complex dependencies where the current conditions can alter the order of tasks or whether some tasks are added or deleted. Throughout the care process, there are documentation and communication requirements with services being delivered by geographically and organizationally separated care providers. In addition, the process must be diligently monitored to ensure compliance with best practices with clinical guidelines and with legal and ethical regulations. Continual evaluation of the processes is needed to improve policy and patient centred care. There are many activities for a single patient and with the many patients that a healthcare system must handle and therefore many sources of errors. A report from 1999 cited in [1] estimated that approximately 98,000 deaths per year in the United States were the result of medical errors and that many of the errors could be traced to faulty processes. Other errors do not lead to death but are costly for the system and adversely affect the patient. Types of medical errors include reasoning errors, errors due to a lack of knowledge, errors due to fatigue and errors due to distraction. Automated decision support can improve the task of complex reasoning. Lack of knowledge includes incomplete information caused by delays in communication or lack of proper access to information. Fatigue and distraction can be traced, in part, to the complexity of the processes involved coupled with limited and strained resources. Information technology can provide solutions by streamlining processes, easing communication and documentation, and helping to provide the most up to date information to clinicians and care providers as they need it. In particular, the web offers many opportunities for instant access to information and knowledge from a variety of sources.

The StFX Centre for Logic and Information [2] was 
established under funding from The Atlantic Canada Opportunities Agency (ACOA), Canada Foundation for Innovation (CFI), and the Nova Scotia Research and Innovation Trust (NSRIT) with the goal of building decision-support through dynamic workflow systems for health care. An interdisciplinary team consisting of researchers and students in computer science, informatics and health related fields have been working closely with clinicians, administrators and other healthcare providers toward developing next generation workflow process and information management systems which can maximize the potential of Canada's emerging electronic health record and improve health services delivery by providing automated decision support. Our goal is a Careflow Management System (CfMS), i.e., a workflow management system for health care that will integrate: (1) traditional workflow management to aid the design, assessment, management, and monitoring of the complex processes, (2) ontologies to structure the data into a usable knowledge base and use the knowledge to guide the workflow, (3) verification tools to ensure processes are designed and executed in accordance with their specifications, (4) high performance computing methods to further aid the handling of large amounts of data and the resulting complex computations in a timely manner, (5) role-specific, interactive, webbased interfaces to meet the needs of a diverse set of end users. Compliance with current messaging standards, such as HL7 V3, to facilitate interoperability is a guiding principle throughout.

In this paper we discuss relevant issues and initial work towards the realization of these goals. The paper is organized as follows. In Section II, we describe the strengths and weaknesses of some existing approaches to managing health care delivery. In Section III, we detail our innovative approach to develop a web-based process and information management system. Section IV presents challenges and related and future work. Finally, Section $\mathrm{V}$ concludes the paper.

\section{CURRENT PROCESS AND INFORMATION Management in Health Care}

In this section we examine three common approaches currently used to improve the management of healthcare systems: clinical guidelines, workflow management systems and electronic health records. We give some background information where needed.

\section{A. Clinical Guidelines}

Clinical guidelines, or protocols, guide decisions by providing criteria regarding diagnosis, management, and treatment in specific areas of healthcare; e.g., post-stroke rehabilitation and diabetes management. Clinical guidelines have been used for thousands of years as a way to pass on the best known medical care practices. The goals are to standardize medical care, to raise the quality of care, and to reduce the risks involved. Clinical guidelines define the most important questions related to clinical practice and identify possible decision options and their outcomes. However, clinical guidelines have traditionally been evaluated and validated though informal methods such as peer reviews. Further, the guidelines themselves are often described using language that may be ambiguous. A formal definition can identify the ambiguity, as well as identify inconsistencies in the guidelines [3].

\section{B. Workflow}

The Workflow Management Coalition provides the following definitions: [4].

A workflow is the automation of a (business) process, in whole or part, during which documents, information or tasks are passed from one participant to another for action, according to a set of procedural rules.

A Workflow Management System (WfMS) is a system that defines, creates and manages the execution of workflows through the use of software, running on one or more workflow engines, which is able to interpret the process definition, interact with workflow participants and, where required, invoke the use of information technology tools and applications.

A process definition is the representation of a business process in a form which supports automated manipulation, such as modeling, or enactment by a workflow management system. The process definition consists of a network of activities and their relationships, criteria to indicate the start and termination of the process, and information about the individual activities, such as participants, associated applications and data, etc.

WfMS are used to identify needs, to reduce waste and duplication of work, to ensure completion of projects on time and in accordance with plans, to improve efficiency, to facilitate documentation, and to create solutions based on the analyzed process requirements. WfMSs can improve efficiency and decrease errors by providing support for modeling, execution and monitoring of workflows. Processes are defined using some (often graphical) modeling language. For example, Fig. 1 shows a simple process with: sequential tasks (Referral then Assess), a choice of tasks (Test 1 or Treatment A), parallel tasks (Treatment B and Treatment C), and an conditional task (Treatment $\mathrm{D}$ depending on Evaluation). Most WfMSs allow the simulation of processes and changes can be made to the model based on the observed results. However, many healthcare processes involve the synchronization of numbers of workflow processes carried out in a distributed setting with dependencies between tasks, agents and resources. The processes must adapt to changes in the patients' situations as well as changes in the system environment, e.g., resource availability. It is difficult and time consuming, to determine the full ramifications of such local changes on the overall process and many processes are extremely time sensitive. Formal verification methods can be used to automatically detect whether the process satisfies requirements both before and after changes. 


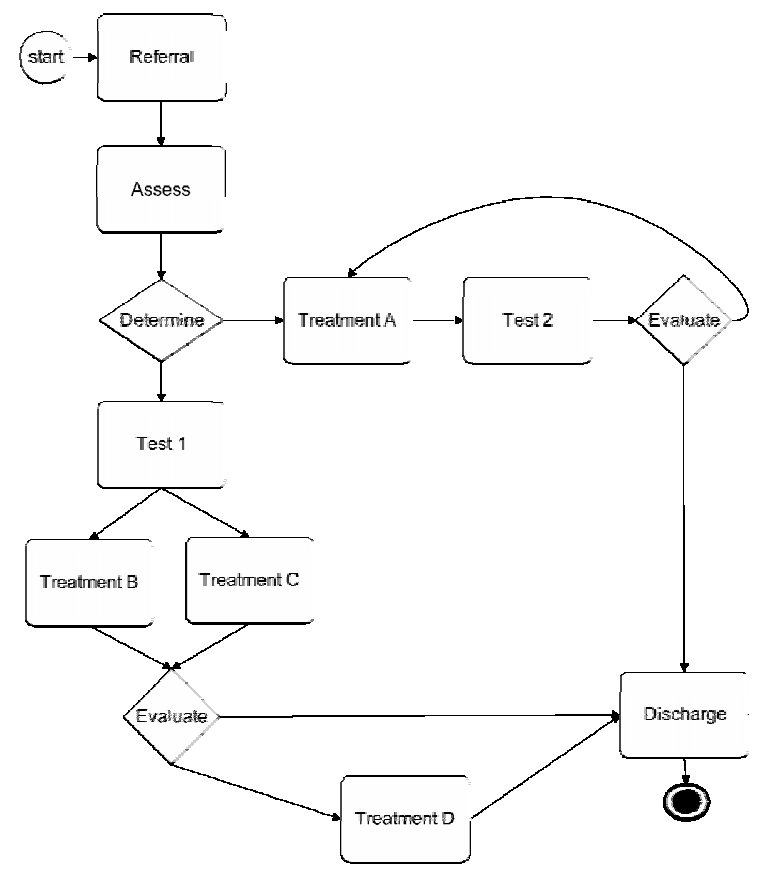

Figure 1. Simple health care process

\section{Electronic Health Records}

Electronic health records (EHR), when available, can greatly enhance communication by facilitating the sharing of information. Canada Health Infoway lists among the benefits of EHRs: reduced wait times, management of chronic care, and improved access to care in rural areas [5]. EHRs alone do not provide these benefits [6]. The EHR must be integrated with effective and efficient means of extracting, organizing, and sharing knowledge in a secure manner. To facilitate this, the information contained in the EHR must be highly structured. Electronic health records do not contain only text documents. They often contain images or video as well. The relevance and relationship these non-textual records have for the patient must be indicated. This is often done using metadata, that is, data about data.

Clinical guidelines, workflow management systems and electronic health records each address the complexity of healthcare processes. While each approach is successful to a degree, an integrated approach, using advanced technological methods such as emerging web technologies, high performance computing, structured knowledge bases and, where possible, automated reasoning, can provide improved, safer, more timely support for healthcare management.

\section{An InNOVATIVE ApPROACH TO CAREFLOW MANAGEMENT}

A Careflow Management System must start with the formalizing of clinical processes and guidelines to provide clear, unambiguous process definitions which will, in turn, permit detailed, automatic verification. The system must be able to seamlessly integrate the emerging EHR and should build on the proven success of current workflow managements systems while adding additional functionality to address the more stringent correctness requirements of healthcare processes. A conceptualization of the system is shown in Fig. 2: it includes a workflow management component, a data management component utilizing EHR and ontologies (see below), and a verification component, along with associated web interfaces for computers, cell phones and other handheld devices. The process definition will be created within the workflow management component and validated and informed by verification of the specifications. The ontology structures the data contained in the EHR and database(s). The verification component uses this structure to identify implicit dependencies as well as the full effects of changes in the process definition.

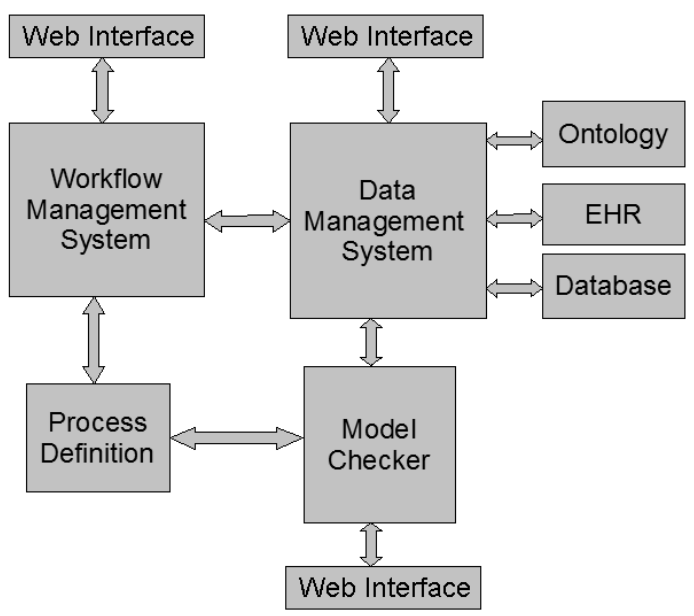

Figure 2. Careflow Management System

\section{A. Knowledge management using Ontologies}

An ontology is a description of a domain which can include all or some of the following [7]:

- objects in the domain;

- $\quad$ properties or attributes of the objects;

- relations between objects;

- properties and relations that evolve over time;

- events that transpire over time and/or can cause other events or states as effects;

- processes in which objects participate;

- states of the world;

- objects that can have parts.

An ontology is a method of structuring knowledge in a usable format to allow reasoning about and sharing of this knowledge. Ontologies have been used extensively in artificial intelligence to describe agents, agent attitudes and information sharing among agents. Ontologies provide an efficient method of managing medical/health and organizational knowledge and can be used to identify 
s minimum data sets for specialized medical domains. Minimum data sets are used to create comprehensive assessment while avoiding collecting data that duplicates or can be derived from data previously acquired. Ontologies can support different views of processes for the diverse participants. An ontology for organizational structure, such as that of a healthcare organization as shown in Fig. 3, can be used to facilitate automatic communication and report generation and routing, a key activity of administrators and policy makers. Having a formalized and structured knowledge base allows the system to be more easily customized for different patients, health authorities or provinces.

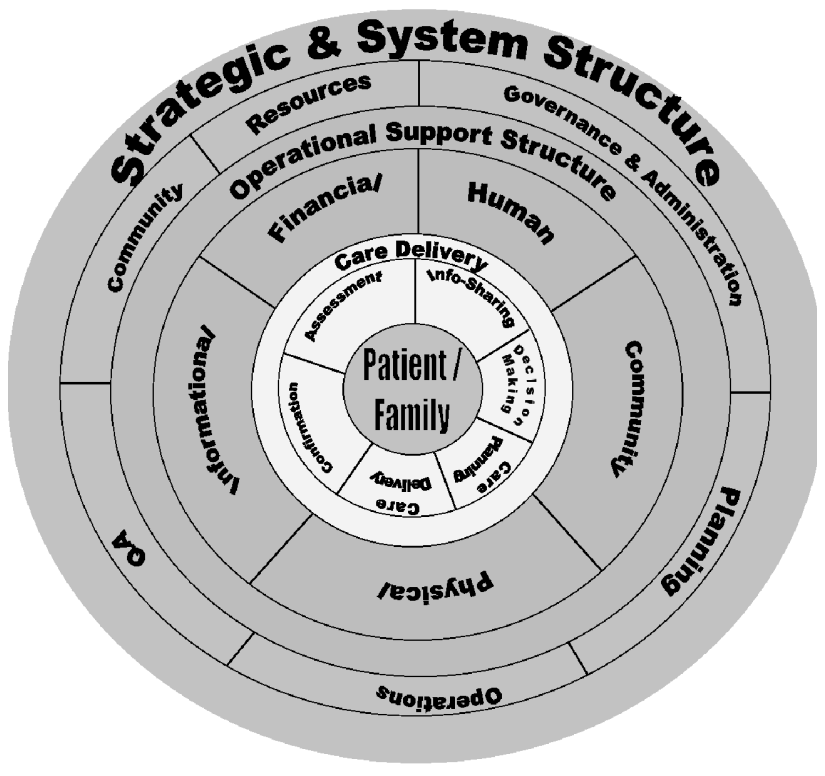

Figure 3. Healthcare system structure

This knowledge of the domain provides metadata that can be utilized to enhance the personalization of the user experience and to build process intelligence into the system. Identification, and use, of this metadata is needed to realize of the promise of the intelligent web. Applications in healthcare include:

- eHealth - the application of information and communications technologies in the health sector, e.g., electronic patient administration and telemedicine [8];

- Health 2.0 - participatory healthcare characterized by the ability to rapidly share, classify and summarize individual health information with the goals of improving health care systems, experiences and outcomes via integration of patients and stakeholders [9];

- Medicine 2.0, Web-based services for health care consumers, caregivers, patients, health professionals, and biomedical researchers, that use Web 2.0 technologies and/or semantic web and virtual reality approaches to enable and facilitate specifically 1) social networking, 2) participation, 3)

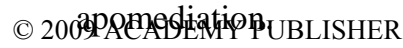

[direct access to expert sources via the web, i.e., there are no intermediaries], 4) openness, and 5) collaboration, within and between these user groups [10].

Ontologies for the medical domain, such as ICNP® (International Classification for Nursing Practices) and SNOMED CT ${ }^{\circledR}$ (Systematized Nomenclature of Medicine - Clinical Terms), as well as numerous drug ontologies have been developed for the electronic exchange of clinical health information. However, these are used primarily as glossaries for terms. Our ontology will be built using the sound scientific principles put forth by the Open Biomedical Ontologies (OBO) Foundry which include [11]:

- $\quad$ The ontology is in, or can be expressed in, a common shared syntax;

- The ontology has a clearly specified and clearly delineated content;

- $\quad$ The ontology must be orthogonal to other ontologies already lodged within OBO;

- The ontologies include textual definitions for all terms.

- Textual definitions will use the genus-species form: An $A \stackrel{\text { def }}{=}$ a B which Cs, where B is the parent of the defined term $A$ and $C$ is the defining characteristic of those Bs which are also As.

Tools, such as Protégé, based on the Web Ontology Language (OWL), can be used to design, build, and analyze ontologies [12]. The Careflow Management System we are developing will enhance decision support by providing the ontology to guide and inform the workflow process using real-time access to dynamic knowledge.

An ontology language provides a way to incorporate intelligence into the system by extracting implicit knowledge from explicit facts in the knowledge base. Ontologies can also increase flexibility through the use of this implicit knowledge to identify the full ramifications of changes to the processes. For example, one aspect of an ontology is the hierarchical structure of the concepts. This allows for the definition of rules for individual cases, classes of cases or all cases to be formulated. Thus, there may be rules for all medications, other rules for pain medications and still more for ibuprofen. For example, specifying that no drug be taken prior to some test implicitly specifies that ibuprofen cannot be taken prior to that test. Other rules are not absolute and may be overridden by knowledgeable professionals; indeed caregivers often deviate from guidelines for various reasons, including when exceptions occur. This must be allowed and the system should capture not only the deviation but the reasons as well. An ontology can encode which rules may be overridden and by whom; this is commonly referred to as privileges. Access control involves another kind of privilege: who has access to which data items. Access privileges are dynamic as patients may grant or withdraw consent to share some or all information relating to their case [13]. Rules for access privileges can be captured in the ontology. Ensuring that patient and family preferences 
for information dissemination are followed is a difficult task. Automatic methods of verification, discussed in the next section, can be applied to provide assurance that information is only accessible by authorized personnel. In fact, verification methods have been used extensively with regards to security and access control.

\section{B. Process Verification}

Verification is the use of mathematical techniques to prove the correctness of an abstract system design. Verification techniques have been used extensively in the design of hardware systems and more recently for software systems as well. One such technique, model checking [14], traverses every possible configuration of a system to determine if the design specifications are always satisfied. A model of a system consists of a description of the initial states of the system along with a description of how the system evolves. Verification of a software system or program consists of proving the correctness of the system or program; that is, proving that the system always meets its specifications. For many systems, appropriately chosen test cases provide sufficient confidence or validation that the system requirements are met. There are many classes of systems, however, for which testing is not sufficient, in particular, systems where errors have catastrophic effects, be it loss of money, time or even human lives. For such so-called "critical" systems, formal methods have been developed including those suitable for automation, such as theorem proving and model checking.

Our work is focused on model checking. Model Checking is an automatic, model-based, propertyverification method [15]. A model checker determines if a specified property is satisfied by the model (or description) of a system and, in general, generates a counter-example if the property is violated. A key feature is that this verification is done fully automatically. Model checking can provide quality assurance that goes beyond that which merely testing or simulation can achieve. While a failed test can conclusively prove the presence of an error, a successful test does not prove the absence of errors. Model checking can prove that a process complies with guidelines every time and not just for the test cases. Model checking can determine, for instance, whether some specified condition always holds or sometimes holds, or never holds in a process. In order to specify more complex timing constraints and other constraints, we employ nonclassical logics, e.g., timed temporal logics, modal logics, logics of beliefs, desires and intentions (BDI), and paraconsistent logics.

Many model checkers use temporal logics which allow the specification of qualitative properties, such as the relative ordering of events. For these logics, time is implicit. Examples of this type of property would be "A patient must be evaluated by an oncologist before beginning chemotherapy," or "A patient must have an assigned Case Manager at all times.” Other temporal logics (timed temporal logics) can be used which allow explicit reference to time. The advantage of using timed temporal logics is that quantitative properties involving time can be specified, e.g., "A patient must have been evaluated by an oncologist within three weeks of beginning chemotherapy.”

Modal logics, such as BDI logics, allow the specification of properties relating to individuals and their views of the processes. This allows local conditions, such as patient issues or particular challenges faced by rural areas, to be incorporated as a guiding factor in the design and execution of patient specific processes. Our patient, Bertha's particular preferences can be captured using BDI logics and used to guide the decisions as much as possible. For example, suppose Bertha does not want a particular care provider to access her records. This preference can be expressed as a BDI logic formula and model checking can determine whether a proposed workflow satisfies this formula.

In addition to this conceptual view of healthcare systems, BDI logics can be used describe and reason about implementations of software systems using agentbased technology. There are many arguments for using agent technology in the design and implementation of systems such as the CfMS we are developing. Health care is highly distributed, involving many concurrent tasks and involves an interdisciplinary team of healthcare providers each with different views of the ongoing care process. Agent-based formalisms can describe this. However, the question of whether the software system has the degree of autonomy usually associated with agent technology is currently being investigated.

Paraconsistent logics provide techniques for reasoning in the face of uncertainty or ambiguity: a common occurrence in a healthcare setting. In [16], we present a prototype next-generation multi-threaded model checker to reason about timed processes in careflows sensitive to patient defined priorities and the goals of the careteam using a temporal logic extended with modalities of beliefs, desires and intentions. The model checker determines whether properties relating to a specified patient's priorities and situation, which is described by an ontological structure, hold for careflows modeled as Timed Colored Petri nets [17].

Model checking does have limitations as it requires the exhaustive search through all possible configurations of a system. This can require a very large amount of memory for even moderately complex systems. In addition this search may take an unreasonable amount time, a critical issue in the very time-sensitive healthcare domain. Many methods have been developed to improve efficiency such as partial state reduction, abstraction techniques, and modular composition. These approaches all seek to reduce the number of states that must be explored. An alternative approach that is emerging is that of high performance computing methods: dividing the model checking problem among multiple processors running in a parallel or distributed environment [18], [19]. Different combinations of these approaches may provide further improvements.

Verification has been successfully used in the design 
of hardware and software systems for many years. In 1992, the verification of a protocol for ensuring data consistency in the IEEE Futurebus+ standard was able to find several previously undetected errors and potential errors in the design of the protocol [20]. Verification has also been used extensively in verifying security protocols, access control protocols and communication protocols. Verification methods have been applied to healthcare as well; in [1], for example, errors were detected in a blood transfusion protocol using model checking.

Workflow management systems generally provide some validation of structural correctness of modeled processes, e.g., that there is a connected path from the start of the workflow to the end of the workflow. However, WfMSs do not allow the verification of more meaningful properties, for example, properties involving complex time dependencies. This leads to workflows being enacted without the certainty of correctness. This often results in errors that need to be handled in an ad hoc manner at runtime and at prohibitive cost [21]. Ad hoc exception handling of errors is unacceptable for workflow for healthcare. Traditional workflow management is typically concerned with efficiency and timing of the workflow. While these are important factors in health care workflow, there is an added factor that the 'work' passing through the workflow includes human patients with needs and preferences that a manufacturing or paper-pushing process does not need to consider. Current systems are sufficient for established, well-defined activities such as manufacturing or processing insurance claims. However, most systems lack the flexibility to adapt to a changing environment in which not all information is known in advance.

One reason for the lack of verification in current workflow systems is that the process definition languages used are not formal enough in the mathematical sense. Much of our work on modeling has been based formally defining the workflow using Petri nets ([32]) and YAWL ([33]) as well as in the input languages of several model checkers.

We can distinguish between static and dynamic verification. Static verification of the careflow model is done to ensure that unsafe behaviors are not permitted or that some desired outcome is always possible in the system design. This can be done early to guide the design of the process. Examples of requirements in health care might be, "Never allow incompatible treatments or drugs", or "Never assign a treatment that has previously failed for that patient.” Dynamic verification during the execution of an instance of the careflow is also important to, for example, determine options from the current position in the workflow that are compatible with the patient's current priorities and situation. By combining real-time monitoring the processes with dynamic verification we aim to improve the overall experience of the patient and improve outcomes. Monitoring consists of observing the flow of a workflow execution or simulation. It involves logging and tracking of aspects of the workflow or workflows. Histories are kept for aggregate data such as average time

(c) 2009 ACADEMY PUBLISHER to complete or maximum wait time for resources. WfMSs typically allow users to indicate what aspects they wish to monitor, or track. Reports generated from this information are then used to guide the reconfiguration or redesign of the workflows. Runtime monitoring can be used to ensure compliance with guidelines or to capture reasons for deviation that may also lead to process redesign. Web technologies offer methods, such as social networking and Really Simple Syndication (RSS) feeds, for instant access to the data needed to monitor the processes and to disseminate any resulting alerts or requests for action.

The CfMS under development will allow patients entered into the system to be followed by diverse but cooperating caregivers through multiple processes using a variety of web-enabled, possibly mobile, devices. The system would ensure that the processes involved complied with all requirements or alert the appropriate parties if an error or possibly undesirable outcome were detected. The system will facilitate speedy and accurate communication, automatic where possible, between the caregivers, administrators, patients, and other concerned parties. Caregivers will have fast, dependable access to the information contained in the system in real-time and be alerted to changes relevant to their concerns. Administrators and policy makers will have instant access to the aggregate data needed to perform their duties. The processes will be transparent; allowing patients to monitor and be as active in the process as they desired and is advisable.

\section{Challenges AND Related WORK}

This paper has described a proposed Careflow Management System being developed as part of an applied research project currently being carried out in close collaboration with local health authorities, university faculty members and students, and industry. To accomplish this we are building on previous research with strong interaction with clinicians and other care providers and applying recent advances in computer science in order to:

1) Model health care processes for monitoring and tracking;

2) Formalize, implement, and verify clinical guidelines and careflow processes;

3) Develop domain ontologies and identifying minimum data sets.

Much of the current research on computer interpretable guideline modeling involves finding a middle ground between a formalism expressive enough to describe all of the aspects of interest but simple enough for nonspecialists to use [22]. Asbru, for instance, defines clinical guidelines and protocols as time-oriented skeletal plans to provide a way to reuse existing domain-specific procedural knowledge while allowing for execution-time flexibility to achieve particular goals [3]. The GuideLine Acquisition, Representation and Execution (GLARE) uses a small set of clearly defined primitives to represent guidelines as different types of actions linked by control relations to define the order of execution [23]. Model checking has been applied to Asbru using SMV and a 
subset of the properties the model checker can verify. Initial work on model checking GLARE by translating the guideline to Promela, the modeling language for the popular SPIN model checker is presented in [24]. The verification for these systems is limited to particular types of simple properties applied to relatively small guidelines with no runtime monitoring or ontology interaction.

The NewGuide Clinical Guideline Management System was developed at the Laboratory for Biomedical Informatics at the University of Pavia, Italy, for acute stroke care and is currently used in two hospitals [25]. It uses three independent modules: (1) a Guideline Management System that provides clinical decision support, (2) an Electronic Patient Record, and (3) a Workflow Management System that provides organizational support. The main focus is on process management, communication management, and organizational learning. The system uses a semiautomatic, knowledge-based approach to error handling but formal verification is not done [25]. The Electronic Patient Record is in a standard data base and can be queried using Structured Query Language (SQL) but does not allow the reasoning available with the query languages for ontologies.

Little-JIL is a graphical language for defining processes that coordinate the activities of autonomous agents and their use of resources during the performance of a task. A model checker for Little-JIL was able to detect errors in a blood transfusion protocol [1] and a chemotherapy process [26]. In [26], it is suggested that the modeling and analyzing of medical processes could benefit from an ontological structure to the domain knowledge but this was not implemented in their study. Initial efforts by our Centre to develop a model checker that interfaces with a medical ontology was presented in [27] and extended in [18].

Developing domain ontologies, especially for a domains as rich as those of healthcare, is a monumental task in itself. It requires close collaboration between developers and clinicians, and other caregivers. Medical ontologies, like SNOMED CT ${ }^{\circledR}$ and ICNP ${ }^{\circledR}$, as well as innovative techniques to merge ontologies without losing knowledge provide a foundation to build upon and will greatly speed up the work [28]. One current initiative of our Centre is to develop a strategy for, and begin the process of, building a minimum data set for hospice palliative care and to link the data set to service delivery and program/policy development [2]. This initiative is being carried out by researchers at StFX, the University of Ottawa, Dalhousie University and others working in close collaboration with healthcare professional including administrators, clinicians and other practitioners.

Other challenges include issues of user adoption and message delivery. Input from frontline caregivers is a continual process in the research conducted at our Centre to ensure that the solutions are in line with caregiver needs. Compliance with existing and emerging standards, such as HL7, is needed to facilitate interoperability with other health information systems, such as the EHR [29]. (c) 2009 ACADEMY PUBLISHER
As noted in [30], medical professionals do not sit at a computer waiting for alerts. We intend to exploit innovative methods of information delivery, such as RSS feeds, that are accessible via web-based mobile devices. For instance, a recent project called ChARMTracker is developing a system using social networks along with instant messaging technologies to provide real-time information to doctors concerning autistic patients [31]. This approach could be applied to any chronic condition. Other web-based delivery methods will also be exploited, such as wikis and podcasts for education of patients and caregivers.

\section{CONCLUSION}

Decisions in health care must be made in a timely manner, with possibly incomplete information, and in a dynamic environment. The decisions carry the added burden of having severe or even fatal consequences of error. Workflow management systems offer a means of reducing human error by organizing and managing the work that must be done and providing support for ensuring that the work is done according to a plan. Formal verification can further reduce errors by ensuring that the plans involved conform to required standards for patient safety. Incorporating a means to go beyond patient safety and in addition reason about patients' preferences offers a means of improving the quality of care and the overall experience of the patients. Current and emerging web- based technologies offer many opportunities for creating a truly collaborative, highly interactive and appropriately responsive care delivery system.

How would our patient, Bertha, benefit from a Careflow Management system such as that described herein? The processes would be transparent, allowing Bertha to monitor and be as active in the process as she desires. This may include subscribing to an RSS feed that updates her as events that concern her occur. Bertha would have access to educational resources, such as a wiki, as well as social networks or blogs that provide support and interaction with other patients, family and caregivers. She would have the ability to interact with healthcare professionals over the web regardless of their physical location. The processes would be verified to ensure: compliance with guidelines and regulations; that conflicting medications are never prescribed; that dependencies are followed; and that information is distributed in a timely manner and only to authorized personnel. Berthas schedule would adapt to changes due to patient specific features such as, missed appointments or evolving preferences.

Caregivers and administrative personnel would reap benefits as well. Documentation would be streamlined and information would be readily available while realtime model checking would ensure that access is limited to those who are authorized and need it. Communication among caregivers would be enhanced; both in speed of distribution and with reduced errors as well as with greater clarity. The time saved would allow more time with the patient. Administrative personnel would have access to 
appropriate data for evaluation, planning/scheduling, and policy development.

\section{ACKNOWLEDGEMENT}

The research described in this paper would not be possible without the extraordinary contributions by the dedicated professionals at the Guysborough Antigonish Strait Health Authority and the St Francis Xavier School of Nursing. Their continued enthusiastic efforts will play a significant role in the successful development of a Webbased Careflow Management System.

\section{REFERENCES}

[1] L. Clarke, Y. Chen, G. Avrunin, B. Chen, R. Cobleigh, K. Frederick, E. Henneman and L. Osterweil, Process Programming to Support Medical Safety: A Case Study on Blood Transfusion. Amherst, MA. (2005)

[2] Centre for Logic and Information. http://logic.stfx.ca/ Antigonish, NS (Last accessed Mar 2009)

[3] M. Marcos, H. Roomans and A. ten Teije and F. van Harmelen, Improving medical protocols through formalisation: a case study. In Proceedings of the Sixth World Conference on Integrate Design and Process Technology. (2002)

[4] Workflow Management Coalition Terminology and Glossary. Technical Report (WFMC-TC- 1011) v3.0. Workflow Management Coalition, Winchester, UK. (1999)

[5] Canada Health Infoway. http://www.infowayinforoute.ca/lang-en/ (Last accessed March, 2009)

[6] 2015: Advancing Canada's Next Generation of Health Care. Canada Health Infoway Report. (2008)

[7] B. Chandrasekaran, R. Josephson and V.R. Benjamins, What are ontologies, and why do we need them? IEEE Intelligent Systems. vol 14, no 1, pp 20 - 26. (1999)

[8] Health Canada: eHealth, http://www.hc-sc.gc.ca/hcssss/ehealth-esante/index-eng.php . (Last accessed Jul 2009)

[9] Health 2.0, http://www.health2con.com/. (Last accessed Jul 2009)

[10] G. Eysenbach, Medicine 2.0: Social Networking, Collaboration, Participation, Apomediation, and Openness, http://www.jmir.org/2008/3/e22/. (Last accessed Jul 2009)

[11] OBO Foundry Principles. http://obofoundry.org/wiki/index.php/OBOFoundry_Principles (Last accessed Jul 2009)

[12] The Protégé Ontology Editor and Knowledge Acquisition System. http://protege.stanford.edu/ (Last accessed December 2008)

[13] Ferris, F.D. and Balfour, H.M. and Bowen, K. and Farley, J. and Hardwick, M. and Lamontagne, C. and Lundy, M. and Syme, A. and West, P.J., A Model to Guide Hospice Palliative Care: Based on National Principles and Norms of Practice. Canadian Hospice Palliative Care Association, Ottawa, ON. (2002)

[14] Clarke Jr., E. M. and Grumberg, O. and Peled, D. A., Model Checking. MIT Press, Cambridge, Massachusetts. (1999)

[15] M.R.A. Huth and M.D. Ryan, Logic in Computer Science: Modelling and Reasoning about Systems. Cambridge University Press, Cambridge, England. (2000)

[16] K. Miller and W. MacCaull, Verification of Careflow Management Systems with Timed BDI $\mathrm{BTL}_{\text {LT }}$ Logic. 3d International Workshop on Process-oriented information systems in healthcare (ProHealth 09)

[17] K. Jensen.: Coloured Petri nets: A high level language for system design and analysis. In: Applications and Theory of Petri Nets, pp. 342-416. (1989)
[18] K. Miller, Timed $B D I_{C} T_{L}$ Verification of Ontology Driven Workflow in a Shared Memory Environment. Master's Thesis. Saint Francis Xavier University. (expected 2009)

[19] H. Wang and W. MacCaull. Distributed-Memory Verification of Real-Time Systems using Explicit-time Description Methods. Submitted to the 38th International Conference On Parallel Processing (ICPP-2009).

[20] E.M. Clarke, The Birth of Model Checking. In 25 Years of Model Checking: History, Achievements, Perspectives, pp 1-26, Springer-Verlag, Berlin, Heidelberg. (2008)

[21] W.M.P. van der Aalst, The Application of Petri Nets to Workflow Management. In: The Journal of Circuits, Systems and Computers, vol 8, no 1, pp. 21-66. (1998)

[22] OpenClinical: Knowledge Management for Medical Care. http://www.openclinical.org/gmmsummaries.html (Last accessed March, 2009)

[23] P. Terenziani, C. Carlini and S. Montani, Towards a Comprehensive Treatment of Temporal Constraints in Clinical Guidelines. in Proc. Ninth International Symposium on Temporal Representation and Reasoning (Time-02), IEEE Computer Society Press, Manchester. (2002)

[24] L. Giordano, P. Terenziani, A. Bottrighi, S. Montani and L. Donzella, Model Checking for Clinical Guidelines: an Agent-based Approach. AMIA 2006, accepted for publication, Washington. (2006)

[25] S. Panzarasa, S. Madde, S. Quaglini, C. Pistarini and M. Stefanelli, Evidence-based careflow management systems: the case of post-stroke rehabilitation. J. of Biomedical Informatics, vol 35, no 2, pp 123-139, Elsevier Science, San Diego, USA. (2002)

[26] S. Christov, B. Chen, G.S. Avrunin, L.A. Clarke,L.J. Osterweil, D. Brown, L. Cassells and W. Mertens, Rigorously Defining and Analyzing Medical Processes: An Experience Report. MoDELS 2007 Workshops, LNCS 5002, pp 118-131, Springer-Verlag, Berlin, Heidelberg. (2008)

[27] J. Dallien, W. MacCaull and A. Tien, Initial Work in the Design and Development of Verifiable Workflow Management Systems and Some Applications to Health Care. In: 3rd Workshop on Agents Applied in Health Care, 5th International Workshop on Model-based Methodologies for Pervasive and Embedded Software, pp. 78-91, MOMPES. (2008)

[28] F. Imam, An Inconsistency Tolerant Approach to Ontology Merging. Master's Thesis. Saint Francis Xavier University. (2008)

[29] H. Jewers, N. Foshay, W. MacCaull, J. Norgrove, and K. Miller, Involving Palliative Care Team Members in Designing Information Technology for Assessment, Communication, and Information Management for PatientCentered Palliative Care. International Symposium on the Terminally Ill (2008)

[30] M. Stefanelli, Careflow Management Systems. OpenClinical Briefing Paper (2002)

[31] A Real ROI from Twitter? The Start of Social Medical Networks. http://blogs.zdnet.com/BTL/?p=18618 (Last accessed Jul 2009)

[32] van der Aalst, W.M.P. and Hofstede, Ter A. H. M.,Workflow Patterns: On the Expressive Power of (Petrinet-based) Workflow Languages. In: Jensen, K.(ed.) Proceedings of the Fourth Workshop on the Practical Use of Coloured Petri Nets and CPN Tools (CPN 2002), pp 1-20. (2002)

[33] van der Aalst, W.M.P., Hofstede, A.: YAWL: Yet Another Workflow Language. QUT Technical report, FIT-TR-200206, Queensland University of Technology, Brisbane, (2002) 
Keith Miller is pursuing a Master of Science in Computer Science Saint Francis Xavier University (StFX) in Antigonish, Nova Scotia. He received a Bachelor of Science in Computer Science from StFX in 2006.

He has been a researcher at the StFX Centre for Logic and Information since 2007. He recently presented a paper, "Verification of Careflow Management Systems with Timed BDICTL Logic," at the 3d International Workshop on Processoriented information systems in healthcare (ProHealth '09) in Ulm, Germany.

Wendy MacCaull received a PhD in Pure Mathematics from MGill University in Montreal, Quebec in 1984. She also received Master of Science degrees in Pure Mathematics from McGill and in Mathematical Biology from Dalhousie University in Halifax.

She is the director of the Centre for Logic and Information at StFX. She has also been a Professor in the StFX Department of Mathematics, Statistics, and Computer Science since 1998. She has published in mathematics, computer science, and health informatics journals. 